\title{
Design of an Axial Electric Pump with Permanent Magnetic Levitation
}

\author{
Fan Zhang ${ }^{\text {a, }}$, Li Zeng ${ }^{\text {a }}$, Zhida Zhu ${ }^{\text {a }}$, Fang Chen $^{\text {a }}$, Jin Sun ${ }^{\text {a }}$, Zhou Suwen ${ }^{\text {a }}$ \\ ${ }^{a}$ College of Mechanical Engineering Yangzhou University, Yangzhou 225127, China \\ *Corresponding Author: fzhang @yzu.edu.cn
}

\begin{abstract}
A new axial electric pump with permanent magnetic levitation was presented. First the basic structure, working principle and characteristic of the axial electric pump are introduced. Then the generation of magnetic suspension force, electromagnetic torque and axial force of the motor pump was analyzed. The research results may provide a theoretical foundation for the electromagnetism design of axial induction motor pump.
\end{abstract}

Keywords: permanent magnet, magnetic levitation, axial electric pump, electromagnetism design.

\section{Introduction}

With the development of the world economy and the increasing of people's living standards, electric pump has entered into people's work and life widely. Compared with other structural pumps, such as plunger pump, centrifugal pump, gear pump, rotary plate pump, etc., the electric pump has compact structure, excellent performance, low vibration and noise, long life and other characteristics. The electric pump is mainly composed of three parts: the pump body, the permanent magnet motor and the outer shell. The working principle of the permanent magnet motor is that after the permanent magnet motor is electrified, the pump body is rotated, and the liquid is sucked from the inlet, then flows through the inner part of the electric pump, and then is pressed out from the outlet to achieve the working control of liquid flow rate and pressure.

The electric pump common uses ordinary bearings. These bearings can reduce the friction and heat phenolmenon in the rotation process, but they cannot completely eliminates the power loss of bearing friction, component wear, component heat and other adverse factors, and it is difficult to achieve high-speed running requirements. So a novel axial electric pump is proposed, which use the permanent magnetic bearings to replace the ordinary bearings for supporting the electric pump. Therefore, in addition to the advantages of ordinary electric pumps, electric pump with magnetic levitation could also eliminate friction and wear of supporting rotor, and has no need of lubrication, no pollution and long service life, so it can improve the speed and output power. Now the magnetic bearings have been successfully applied to high-speed and ultra-high-speed rotary machine, such as ultra-high speed camera, cutting machine and faster centrifuge. It has also been widely applied to centrifugal compressors, pumps, driving motors, steam turbines and gas turbines ${ }^{[1]}$.

\section{Basic Structure of the Axial Electric Pump with Permanent Magnetic Levitation}

The axial electric pump with permanent magnetic levitation is mainly composed of permanent magnetic bearings, axial motor, pump, mechanical seal devices and so on, which is shown in Figure 1. The permanent magnet bearings support the rotating shaft, and the axial motor outputs the torque, which is composed of rotor shaft, rotor disc and two stators. Two stators are installed symmetrically on both sides of the rotor disc. The rotor disc is shown in Figure 2, and the stator is shown in Figure 3.

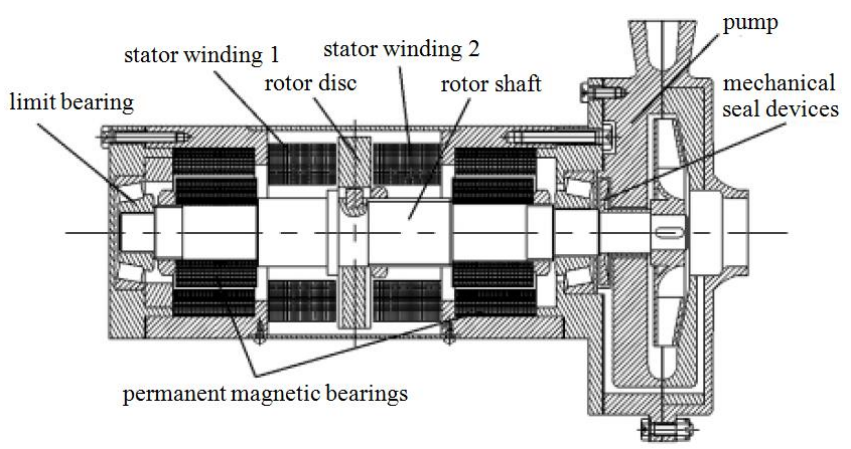

Fig. 1 structure profile of the axial electric pump with permanent magnetic levitation 


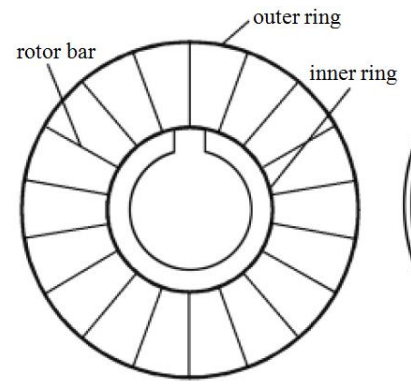

Fig. 2 the rotor disc

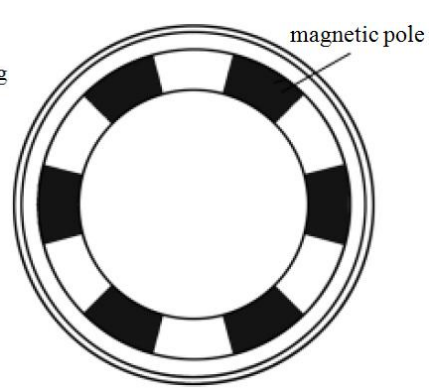

Fig. 3 the stator

\section{Mechanism Analysis of the Axial Electric Pump with Permanent Magnetic Levitation}

\subsection{The Radial Force and Axial Force of Permanent Magnetic Bearings}

The axial electric pump with permanent magnetic levitation uses two pairs of permanent magnet bearings to achieve radial support. According to the literature 2, the selection of axial magnetized permanent magnet bearings is better than radial magnetized permanent magnetic bearings, and permanent magnetic bearings can provide an axial force by biased the permanent magnetic ring pairs. The permanent magnetic bearing structure is shown in Figure 4.

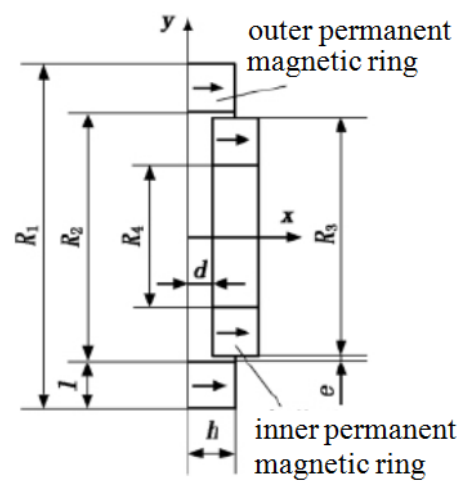

Fig. 4 structure diagram of permanent magnetic bearings

If the radial permanent magnet bearings have axial displacement, and at the same time the radial displacement occurs. Because the radial clearance between permanent magnet rings is no longer uniform, the radial force and axial force can be calculated by magnetic charge method. While any point magnetic charge on the inner surface of the outer magnetic ring and any point magnetic charge on the outer surface of the inner magnetic ring are selected, the interaction force between the two magnetic charges is ${ }^{[3]}$ :

$$
d F=\frac{\left(\sigma_{1} r_{1} d \beta d r_{1}\right) \cdot\left(\sigma_{2} r_{3} d \alpha d r_{3}\right)}{4 \pi \mu_{0}|r|^{3}} \cdot r
$$

Where $\sigma_{1}$ and $\sigma_{2}$ are the magnetic charge density on the end surface of the outer magnetic ring and the inner magnetic ring. $\alpha$ and $\beta$ are the polar coordinates of any point charge on the end surface 1 and $3 . \mu_{0}$ is vacuum permeability. $r_{1}$ is the radius vector of any point on the end surface 1 of the outer magnetic ring, and $r_{3}$ is the radius vector of any point on the end surface 3 of the inner magnetic ring. $R_{1}$ and $R_{2}$ are the outer diameter and inner diameter of the outer magnetic ring, and $R_{3}$ and $R_{4}$ are the outer diameter and inner diameter of the inner magnetic ring. $d$ is the offset. $e$ is the radial clearance. $h$ is the width of ring. $r$ is the radius vector between two point magnetic loads.

The axial coordinate axis is selected as the $\mathrm{X}$ axis, and the projection in the direction of the $\mathrm{X}$ of the force between the end surface 1 of the outer magnetic ring and the end surface 3 of the inner magnetic ring is $F_{13}^{x}$, which is calculated by the formula 2 .

$$
F_{13}^{x}=\frac{\sigma_{1} \sigma_{2}}{4 \pi \mu_{0}} \int_{0}^{2 \pi} \int_{0}^{2 \pi} \int_{R_{2}}^{R_{1}} \int_{R_{4}}^{R_{3}} \frac{-x_{0} r_{1} r_{3}}{\left|r_{13}\right|^{3}} d \alpha d \beta d r_{1} d r_{3}
$$

Where $\left|r_{13}\right|=\sqrt{\left(-x_{0}\right)^{2}+\left(e \cos \theta+r_{3} \cos \alpha-r_{1} \cos \beta\right)^{2}+(e \sin \theta+}$

$\overline{\left.r_{3} \sin \alpha-r_{1} \sin \beta\right)^{2}}, x_{0}$ is the axial displacement of the inner magnetic ring to the outer magnetic ring. $\theta$ is the angle that the inner magnetic ring deviates from the outer magnetic ring in the radial coordinate axis Y. $r_{13}$ is the radius vector from 1 to 3 .

In the same way, the projection in the direction of the $\mathrm{X}$ of the force between the end surface 1 of the outer magnetic ring and the end surface 4 of the inner magnetic ring is as following:

$$
F_{14}^{x}=\frac{\sigma_{1} \sigma_{2}}{4 \pi \mu_{0}} \int_{0}^{2 \pi} \int_{0}^{2 \pi} \int_{R_{2}}^{R_{1}} \int_{R_{4}}^{R_{3}} \frac{-\left(l+x_{0}\right) r_{1} r_{4}}{\left|r_{14}\right|^{3}} d \alpha d \beta d r_{1} d r_{4}
$$

Where $r_{4}$ is the radius vector of any point on the end surface 4 of the inner magnetic ring. $r_{14}$ is the radius vector from 1 to 4 .

The projection in the direction of the $\mathrm{X}$ of the force between the end surface 2 of the outer magnetic ring and the end surface 4 of the inner magnetic ring is as following:

$$
F_{23}^{x}=\frac{\sigma_{1} \sigma_{2}}{4 \pi \mu_{0}} \int_{0}^{2 \pi} \int_{0}^{2 \pi} \int_{R_{2}}^{R_{1}} \int_{R_{4}}^{R_{3}} \frac{-\left(l-x_{0}\right) r_{2} r_{3}}{\left|r_{23}\right|^{3}} d \alpha d \beta d r_{2} d r_{3}
$$

Where $r_{2}$ is the radius vector of any point on the end surface 
2 of the outer magnetic ring. $r_{23}$ is the radius vector from 2 to 3 .

The projection in the direction of the $\mathrm{X}$ of the force between the end surface 2 of the outer magnetic ring and the end surface 4 of the inner magnetic ring is as following: $F_{24}^{x}=F_{13}^{x}$.

When the axial magnetization is magnetized, the magnetic force provided by the permanent magnetic bearings is the algebraic sum of the interaction forces between the 4 ends. The projection force in the direction of the $\mathrm{X}$ is:

$$
F_{a}=F_{13}^{x}+F_{24}^{x}-F_{14}^{x}-F_{23}^{x}=2 F_{13}^{x}-F_{14}^{x}-F_{23}^{x}
$$

\subsection{Electromagnetic Torque and Axial Magnetic Levitation Force Produced by the Magnetic Levitation Motor}

(1) electromagnetic torque

If the current frequency in the stator winding is $f$, and the angular frequency $\omega=2 \pi f$, then the electromagnetic torque $T_{d}$ could be calculated with the equivalent circuit:

$$
T_{d}=\frac{P_{d}}{\omega}=\frac{1-s}{\omega}\left(I_{2}^{\prime}\right)^{2} \frac{R_{2}^{\prime}}{s}=\frac{\left(I_{2}^{\prime}\right)^{2} R_{2}^{\prime}}{s \omega_{s}}
$$

Where $P_{d}$ is the power of each phase; $R_{2}^{\prime}$ is the rotor resistance; $I_{2}^{\prime}$ is the rotor current. $s$ is the slip ratio. $\omega_{s}$ is the synchronous angular frequency. According to the de Venan equivalent circuit and the partial voltage principle, $\left(I_{2}^{\prime}\right)^{2}$ can be obtained ${ }^{[4]}$.

(2) axial magnetic attraction force

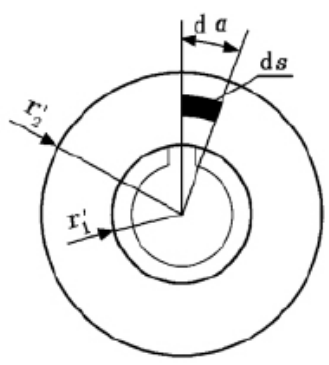

Fig. 5 calculation diagram of axial magnetic attraction force

Under the hypothetical condition ${ }^{[5]}$, the small area $d s$ on the disc is taken as shown in Figure 5. Then the axial magnetic attraction force of the rotor is as following.

$$
F=\int_{s} \frac{B^{2}}{2 \mu_{0}} d s=\frac{1}{2 \mu_{0}} \int_{r_{1}^{\prime}}^{r_{2}^{\prime}} \int_{0}^{2 \pi} B^{2} r^{\prime} d r^{\prime} d \alpha=\frac{r_{2}^{\prime 2}-r_{1}^{\prime 2}}{4 \mu_{0}} \int_{0}^{2 \pi} B^{2} d \alpha
$$

Where $r_{1}^{\prime}$ and $r_{2}^{\prime}$ are the inner and the outer diameter of rotor disc. $r^{\prime}$ is the radius of the position of the small area on the disc. $B$ is the flux density through $d s$.

When the rotating magnetic potential of the base wave in the stator air gap is produced ${ }^{[6]}$, the axial magnetic attraction force is obtained.

$$
F=\frac{\left(r_{2}^{\prime 2}-r_{1}^{\prime 2}\right) \mu_{0} m_{1}^{2} N_{1}^{2} I_{1}^{2}}{\pi \delta^{2}}
$$

Where $m_{1}$ is the number of phases, the $N_{1}$ is the turn number, the $I_{1}$ is the current, and the $\delta$ is the axial gap between the rotor disc and the stator.

\section{Conclusions}

On the basis of the existing magnetic levitation electric pump, the design and analysis of the permanent magnetic levitation axial electric pump. The following conclusions are drawn:

(1)the passive magnetic levitation mode of permanent magnetic bearings is used, and the structure is simple and easy to control.

(2) the axial size of the motor is reduced by axial motor, and it is convenient to be used in the case of small axial space.

(3) when the axial rotor size, axial force and stator winding with the number, number of turns and the excitation current is proportional to the square of.

(4) both sides of the rotor disk have the same axial magnetic force on the rotor, so it can ensure the rotor shaft's stable suspension in the axial direction under the axial support of the permanent magnet bearing..

\section{Acknowledgment}

Thank the China Natural Science Foundation for this project, Item Number: 51375427, 51475409. Thank the Jiang Su Natural Science Foundation for this project, Item Number: BY2014117-08, BY2015061-04. Thank the Yangzhou city - Yangzhou University of Science and Technology Cooperation Program Funds, Item Number: SXT20140041, YZ2016244, YZ2016255. Thank the Research Project of State Key Laboratory of Mechanical System and Vibration, Item Number: MSV201810. 


\section{References}

(1) Hou Shuhong, Jarl Maimaiti: "Development and Prospect of the disc motor in China". Micro and Special Motor, No. 4, pp. 30 - 33, 1998.

(2) $\mathrm{Xu}$ Kaiguo: "Research and design of rotor of permanent maglev wind generator", Wuhan University of Technology, pp. 14 - 15, 2008.

(3) Zhu Meili: "Design and mechanical characteristics analysis of permanent magnetic bearings", Xian University of Technology, pp. 34 - 36, 2008.

(4) Jiang Haoxian, Liang Feng: "Disc type axial magnetic induction motor", China Journal of Electrical Engineering, Vol. 8, No. 2, pp. 56 - 63, 1988.

(5) Gu Qishan, Hou Shuhong: "Design and calculation of magnetic circuit of disk type induction motor", Medium and small motor, Vol. 21, No. 1, pp. 17 - 20, 1994.

(6) Li Da, Ke Jian, Deng Bin: "Research on integrated motor pump device", Mechanical Research and Application, Vol. 20, No. 2, pp. 55-56, 2007. 